\title{
High-resolution IR and radio observations of AGB stars
}

\author{
G. Perrin ${ }^{1}$, W. D. Cotton ${ }^{2}$, R. Millan-Gabet ${ }^{3}$, and B. Mennesson ${ }^{4}$ \\ ${ }^{1}$ LESIA, Observatoire de Paris, CNRS, UPMC, Université Paris-Diderot, Paris Sciences et Lettres Research University, \\ 5 place Jules Janssen, 92195 Meudon, France \\ e-mail: guy .perrin@obspm. fr \\ 2 National Radio Astronomy Observatory, 520 Edgemont Road, Charlottesville, VA 22903-2475, USA \\ 3 California Institute of Technology, NASA Exoplanet Science Institute, Pasadena, CA 91125, USA \\ 4 Jet Propulsion Laboratory, California Institute of Technology, 4800 Oak Grove Drive, Pasadena, CA 91109, USA
}

Received 4 October 2014 / Accepted 20 January 2015

\begin{abstract}
Aims. We present the results of observations with interferometers of a sample of pulsating asymptotic giant branch (AGB) stars in the infrared and at radio wavelengths. The goal of these observations is to explore the extended stellar atmospheres and to establish links between the spatial scales of molecular envelopes and of the dust shell. This is the key to better understand the process of dust formation and therefore of mass loss.

Methods. We used the ESO VLTI/MIDI interferometer in the $N$ band, the Keck Interferometer in the $K$ band, and NRAO VLBA observations of $\mathrm{SiO}$ masers at $7 \mathrm{~mm}$ wavelength of a sample of AGB stars: U Ari, W Cnc, RX Tau, RT Tau, RT Aql, S Ser, and $\mathrm{V}$ Mon. The various instruments probe different altitudes of the atmosphere of the AGB stars. They are sensitive to regions below the silicate dust condensation distance and provide the opportunity of finding hints about how dust and its precursors form in the extended atmosphere of an AGB star. The $K$-band observations are sensitive to water and carbon-monoxyde vapors. Unfortunately, we were only able to observe $\mathrm{S}$ Ser in this wavelength range.

Results. We find a ratio of 2.2 between the molecular envelope radius and the photospheric size, which is consistent with previous results. The $\mathrm{N}$-band observations are mostly sensitive to vapors of $\mathrm{SiO}$ and water and to dust (alumina and silicate). The silicate dust shell is fully resolved, and no precise parameters can be deduced from the $N$-band observations other than a spatial extension of at least $12-16 R_{\star}$ for our sample. The sizes found for the $\mathrm{SiO}$ region are consistent with the radii of the $\mathrm{SiO}$ maser rings provided by the VLBA observations. The sizes of the alumina and water vapor regions are systematically found to be larger. There is clear evidence that $\mathrm{SiO}$ is absent from regions farther from the star where silicate dust condenses.

Conclusions. These observations support a possible scenario in which $\mathrm{SiO}$ is adsorbed by species such as corundum. An alternative explanation could be that $\mathrm{SiO}$ has chemically disappeared at this range of distances.
\end{abstract}

Key words. stars: atmospheres - stars: AGB and post-AGB - stars: mass-loss - masers - techniques: interferometric - infrared: stars

\section{Introduction}

Asymptotic giant branch (AGB) stars have a few solar masses and have reached the end of their lives; the inner core of the star has collapsed, the outer envelope has become very extended and is in the process of losing its mass to the interstellar medium through pulsational instabilities. In the cool, extended envelopes of these stars, molecules form and condense into dust. In oxygen-rich stars, molecular masers appear, especially $\mathrm{SiO}$, $\mathrm{H}_{2} \mathrm{O}$, and $\mathrm{OH}$ at increasing distance from the photosphere (Reid \& Moran 1981). The $\mathrm{SiO}$ masers occur within a few stellar radii of the stellar surface between the hot molecular inner envelope and the cooler region where the (silicate) dust forms; see Reid \& Menten (1997), Danchi et al. (1994). The detailed mechanics driving mass loss is not well understood; nor is the reason why many planetary nebulæ are very asymmetric while their AGB progenitors appear circularly symmetric. Recent findings on the closest Mira-type star L2 Puppis may shed light on new directions to investigate this (Kervella et al. 2014).

Perrin et al. (2004) observed a number of Mira variables between 2.2 and $3.6 \mu \mathrm{m}$ and derived large variations of the apparent size with wavelength. They interpreted the variation in size as being due to a molecular envelope containing $\mathrm{H}_{2} \mathrm{O}$ and a wavelength-dependent opacity.
Ohnaka et al. (2005) observed the Mira variable RR Sco at 2.2 and $10 \mu \mathrm{m}$ using the VLTI and modeled the star with a photosphere surrounded by an optically thick envelope of $\mathrm{H}_{2} \mathrm{O}$ and $\mathrm{SiO} 2.3$ times the photospheric size and surrounded by an optically thin dust shell with an inner diameter of seven to eight times the photospheric size. Danchi et al. (1994) also found considerable variations in the ratio of the size of the inner dust shell to the size of the photosphere at $11 \mu \mathrm{m}$. However, Cotton et al. (2004) found a fairly small scatter of the ratio of the $\mathrm{SiO}$ maser ring diameter to the apparent size at $2.2 \mu \mathrm{m}$ around a value of approximately 2 . This was confirmed by observations of S Orionis by Wittkowski et al. (2007). The relation of the $\mathrm{SiO}$ maser ring diameter to the inner size of the dust shell remains unclear.

In this paper we report observations using the ESO VLTI/MIDI $10 \mu \mathrm{m}$ interferometer, the Keck Interferometer at $2.2 \mu \mathrm{m}$, and NRAO/VLBA observations of $\mathrm{SiO}$ masers at $7 \mathrm{~mm}$ wavelength. A combination of these results places strong constraints on the size and nature of the molecular envelope.

\section{Near-infrared}

The near-infrared wavelength range is primarily sensitive to the photosphere and to the close environment of the star. Mennesson et al. (2002) and Perrin et al. (2004) showed that the opacity 
of a putative envelope at about $2 R_{\star}$ cannot be neglected in order to interpret visibility curves. Observations in specific narrow-band filters pointed to $\mathrm{CO}$ and $\mathrm{H}_{2} \mathrm{O}$ vapors as potential major contributors to the opacity in $K$ band, with water vapor being the main contributor. Spectrally dispersed observations in the near-infrared confirmed this finding (Eisner et al. 2007; Wittkowski et al. 2008; Le Bouquin et al. 2009). At the lowest opacity (in $J$ and $H$ bands), the impact of the envelope is reduced and the diameter of the object is close to that of the photosphere.

\section{Mid-infrared}

A few oxygen-rich Miras have been measured in the midinfrared. Ohnaka et al. (2005) found an envelope containing $\mathrm{SiO}$ and water vapor at 2.3 photospheric radii in RR Sco, which was well modeled with a temperature of $1400 \mathrm{~K}$. Likewise, Wittkowski et al. (2007) found an envelope of $\mathrm{Al}_{2} \mathrm{O}_{3}$ around $\mathrm{S}$ Ori at about 2 stellar radii above the stellar photosphere and just inside the $\mathrm{SiO}$ maser emission ring. Onaka et al. (1989) $\mathfrak{x}$ detected alumina around all stars in a sample of $109 \mathrm{M}$ Mira stars. It is very tempting to compare the mid-infrared molecular envelopes found around Miras to the MOLspheres found around the red supergiants $\mu$ Cep and Betelgeuse to account for the warm water vapor opacity (Tsuji 2000). Perrin et al. (2007) showed that Betelgeuse contains at least SiO, water vapor, and alumina, and Verhoelst et al. (2006) hypothesized alumina dust - whose condensation temperature is high - to be a nucleation site for silicate dust, hence providing a scenario for how a dust flow could be created despite the lack of sustained large amplitude pulsations.

\section{$\mathrm{SiO}$ masers}

Circumstellar $\mathrm{SiO}$ masers tend to occur in clumpy, partial rings centered on the central star (Diamond et al. 1994). A long path through relatively constant (radial) velocity gas is needed for the masers to develop the necessary gain to be visible. This condition is most often met for lines of sight tangent to the masing layer in regions of relatively low turbulence. Turbulence will reduce the path length at a given velocity, which limits the gain, hence brightness, of the corresponding maser spot. The resulting maser spots tend to appear in a partial ring. These maser rings mark the outer layers of the molecular envelope before the $\mathrm{SiO}$ condenses onto grains, and the rings provide a sensitive probe of the gas dynamics in this region.

\section{Near-IR observations and data reduction}

Observations of S Ser were made on UT 2007 July 03 at the Keck Interferometer (KI, Colavita et al. 2013). We used a low spectral dispersion mode, which provides 42 spectral channels across the near-infrared $K$ band $\left(\lambda_{0}=2.18 \mu \mathrm{m}, \Delta \lambda=0.4 \mu \mathrm{m}\right.$, $R=200$, see Eisner et al. 2007). The width of each spectral channel is therefore $\sim 93 \AA$. The average projected length and position angle of the KI baseline was $79.5 \mathrm{~m}$ and $23.0^{\circ}$. The calibrator stars used were HD141377 $(V=6.9, K=2.5$, K5III, $\theta=2.1 \pm 0.9$ mas $)$ and HD150012 $(V=6.3, K=5.3$, F5IV, $\theta=0.4 \pm 0.1$ mas); where their angular diameters $\theta$ have been estimated by fitting a stellar photosphere (approximated by a blackbody) to the measured spectral energy distribution ${ }^{1}$;

\footnotetext{
1 fBol module of the getCal package; http://nexsciweb.ipac. caltech. edu/gcWeb/
}

these estimates agree well with those produced by the surface brightness relations of Barnes et al. (1978).

Reduction and calibration of the interferometer data were made using standard packages provided by the $\mathrm{NExScI}^{2}$. Two independent observations of S Ser yield $V^{2}$ that agree to better than $10 \%$. Consistent with project documentation describing the performance of the KI instrument, we added an external error of 0.03 to the formal statistical errors in the absolute calibration of $V^{2}$. The two measurements have been averaged into a single one, and errors have been reduced using the same procedure as for the MIDI data, as explained in the next section. They are presented in Fig. 1.

\section{Mid-infrared data}

\subsection{Observations and data reduction}

The mid-IR data were obtained in the $8-13 \mu \mathrm{m}$ band with the MIDI instrument of the VLTI (Leinert et al. 2003) in service mode between 11 November 2006 and 3 March 2007. The $1.80 \mathrm{~m}$ auxiliary telescopes of the VLTI were used for these observations, giving access to baselines ranging from $38 \mathrm{~m}$ up to $130 \mathrm{~m}$. The fringes were dispersed using a prism at a spectral resolution of 30 . The entire data reduction made use of the software developed by the Paris Observatory for MIDI, which we previously used in Perrin et al. (2007). Beams were not spatially filtered so that the uncorrelated flux is expected to be constant, but varies as a result of thermal background fluctuations in this wavelength range. Despite the relatively small pupils in this wavelength range, coherence fluctuates. Both effects induce visibility instabilities of several percents from one acquisition to another and hinder the measuring the uncertainties because these are not detected or modeled by the reduction software. We applied the method presented in Perrin et al. (2007) to derive the most realistic error bars and to reduce the impact of biases as best possible. Data were binned as a function of baseline for a given source. The final visibility error bars were adjusted so that errors are representative of the scatter inside bins. Unresolved calibrator stars were chosen near the sources and within the sensitivity range of MIDI. Whenever possible, they were selected from the list of spectral calibrators assembled by Cohen et al. (1999) to serve as primary spectral calibrators. The list of averaged observations is given in Table 1. All sources but S Ser have been observed at different spatial frequencies, which allows us to explore how the visibilities vary with spatial frequency, that is, to explore the source spatial profile. Some of the observations spanned a period of two months, but it has been assumed that the source did not change much across this period of time as the pulsation period is on the order of a year. All observations were made with essentially a single baseline azimuth, and it was therefore not possible to explore deviations from spherical symmetry.

\subsection{Spectrum calibration}

Since the sources are variable, the spectra obtained with MIDI for uncorrelated flux are extremely valuable for modeling the mid-infrared visibilities. We calibrated the spectra using the nearest interferometric calibrators (as spectral calibrators) for

\footnotetext{
2 i.e., Kvis and $n b$ Calib;

http://nexsci.caltech.edu/software/KISupport/
} 

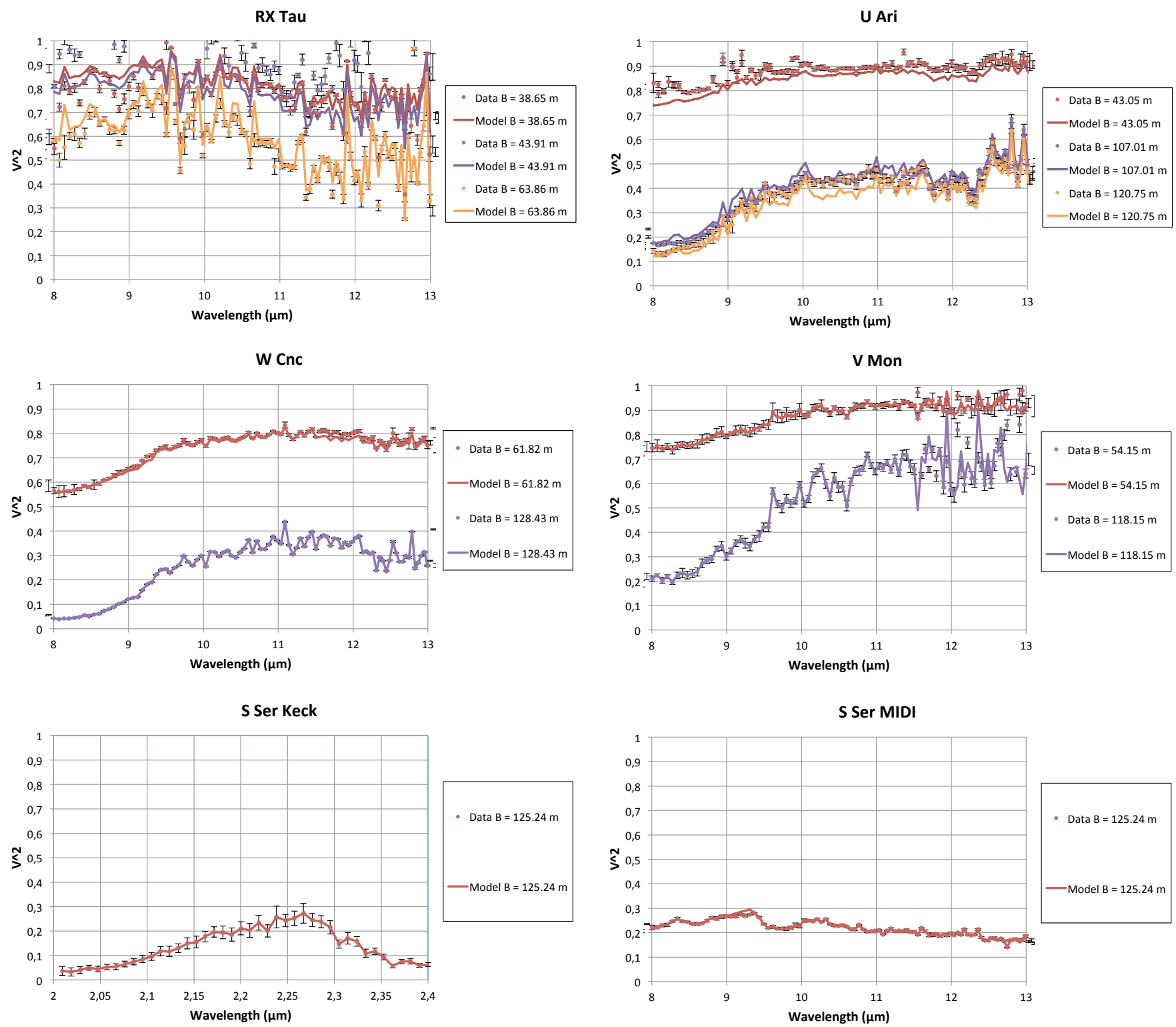

Fig. 1. Measured and modeled mid- and near-IR squared visibilities.

which published calibrated spectra were available. For W Cnc, V Mon, and S Ser we used 6 Leo, 30 Gem, and 110 Vir as spectral calibrators, respectively. Their spectra were published by Cohen et al. (1999). For U Ari we used $\alpha$ Ceti, whose calibrated spectrum was published by Engelke et al. (2006). Last, for RX Tau we used the IRAS LRS spectrum for 119 Tau (Beichman et al. 1988). Since two spectra are measured by MIDI (one per beam), a statistical accuracy of a few percent can be assessed for the spectra. However, no estimate of possible systematic errors could be derived because only one primary spectral calibrator was available for each source. A conservative estimate of the final accuracy of the spectrum is probably on the order of $10 \%$. The spectra are displayed in Fig. 2.

\section{Modeling infrared visibilities}

The principle is to use the simple spherically symmetric star+envelope model presented in Perrin et al. (2004) to derive the geometrical parameters of the molecular environment

around the central source and to compare them with the $\mathrm{SiO}$ maser measurements.

\subsection{Normalization of MIDI visibilities and inner radii of dust shells}

We modeled the MIDI visibilities in different steps. The sources are known to be surrounded by a dust envelope because of an infrared excess, and it is therefore necessary to take this into account to avoid misinterpreting the MIDI visibilities. For sources with visibilities at different baselines (all but S Ser), visibilities were modeled with a simple model first: a resolved environment and a central uniformly bright disk:

$V(B / \lambda)=A(\lambda) \times\left|\frac{2 J_{1}(\pi(\lambda) B / \lambda)}{\pi(\lambda) B / \lambda}\right|$,

where $A$ is the fractional flux of the central object and is independent of baseline $B$. The $A(\lambda)$ factors vary across the $N$ band: RX Tau, 80-100\%; U Ari, 70-100\%; V Mon, 80-100\%; and 
Table 1. Inrafred observations.

\begin{tabular}{|c|c|c|c|c|c|c|c|}
\hline Star & Instrument & Date & Mean UT time & $\begin{array}{l}\text { Mean projected } \\
\text { baseline }(\mathrm{m})\end{array}$ & $\begin{array}{c}\text { Mean azimuth } \\
\left.{ }^{\circ}{ }^{\circ}\right)\end{array}$ & $\begin{array}{r}\text { Period }^{b} \\
\text { (days) }\end{array}$ & Visual phase ${ }^{b}$ \\
\hline U Ari & MIDI & $\begin{array}{l}13 \text { November } 2006 \\
\text { 09 December } 2006 \\
\text { 09 December } 2006\end{array}$ & $\begin{array}{l}01: 53: 02 \\
01: 28: 07 \\
02: 25: 08\end{array}$ & $\begin{array}{l}43.05 \\
107.01 \\
120.75\end{array}$ & $\begin{array}{l}80^{\circ} 56 \\
67^{\circ} 55 \\
66^{\circ} 79\end{array}$ & 372 & $\begin{array}{l}0.03 \\
0.10 \\
0.10\end{array}$ \\
\hline W Cnc & MIDI & $\begin{array}{l}20 \text { December } 2006 \\
07 \text { January } 2007^{c} \\
07 \text { March } 2007^{c}\end{array}$ & $\begin{array}{l}07: 54: 05 \\
07: 29: 34 \\
04: 23: 32\end{array}$ & $\begin{array}{c}61.82 \\
126.90 \\
129.99\end{array}$ & $\begin{array}{l}77^{\circ} 72 \\
66^{\circ} 36 \\
61^{\circ} 96\end{array}$ & 392 & $\begin{array}{l}0.99 \\
0.04 \\
0.19\end{array}$ \\
\hline RX Tau & MIDI & $\begin{array}{l}13 \text { November } 2006 \\
13 \text { November } 2006 \\
19 \text { December } 2006\end{array}$ & $\begin{array}{l}02: 52: 21 \\
03: 18: 20 \\
03: 58: 14\end{array}$ & $\begin{array}{l}38.65 \\
43.91 \\
63.86\end{array}$ & $\begin{array}{l}72^{\circ} 46 \\
73^{\circ} 73 \\
73^{\circ} 09\end{array}$ & 334 & $\begin{array}{l}0.29 \\
0.29 \\
0.39\end{array}$ \\
\hline V Mon & MIDI & $\begin{array}{l}12 \text { November } 2006^{d} \\
13 \text { November } 2006^{d} \\
07 \text { January } 2007\end{array}$ & $\begin{array}{l}05: 53: 06 \\
05: 48: 56 \\
06: 39: 04\end{array}$ & $\begin{array}{c}54.17 \\
54.14 \\
118.15\end{array}$ & $\begin{array}{l}68^{\circ} 06 \\
68^{\circ} 04 \\
61^{\circ} 48\end{array}$ & 334 & $\begin{array}{l}0.22 \\
0.23 \\
0.39\end{array}$ \\
\hline S Ser & $\begin{array}{l}\text { MIDI } \\
\text { Keck }\end{array}$ & $\begin{array}{l}\text { 06 March } 2007 \\
\text { 03 July } 2007\end{array}$ & $\begin{array}{l}09: 18: 16 \\
09: 10: 52\end{array}$ & $\begin{array}{l}125.24 \\
79.73 \\
\end{array}$ & $\begin{array}{l}65^{\circ} 85 \\
23^{\circ} 76 \\
\end{array}$ & 371 & $\begin{array}{l}0.55 \\
0.87\end{array}$ \\
\hline
\end{tabular}

Notes. ${ }^{(a)}$ Counted positive from east to north; ${ }^{(b)}$ computed using the photometric data of the AFOEV at http://cdsarc.u-strasbg.fr/ afoev/; ${ }^{(c)}$ baseline data were averaged together; ${ }^{(d)}$ baseline data were averaged together.

W Cnc, 40-100\%. The lowest values are reached around $10 \mu \mathrm{m}$ as is typically expected for silicate dust. In addition, accounting for the incoherent flux led to very good fits of the central object, which confirms that the visibilities can be modeled with two components, one of which is fully resolved. As a consequence, the visibilities were systematically normalized by dividing by the $A(\lambda)$ flux ratio factor to remove the signature of the external dusty envelope and derive information about the central disk. This process could not be applied to S Ser, and the raw visibilities were therefore used with some unavoidable inaccuracies in the modeling. The processed data used for modeling are presented in Fig. 1.

Our mid-infrared data therefore contain some information about the silicate dust shells around the stars of our sample. Danchi et al. (1994) found the inner radius of the shell located at at $3-5 R_{\star}$, probably the distance at which silicate dust forms. This conclusion was based on assumptions about photospheric radii. These assumptions can be updated with the more recent works on the interpretation of the mid- and near-infrared visibility curves. Mennesson et al. (2002) and Perrin et al. (2004) showed that a molecular envelope at about 2 photospheric radii has to be taken into account to interpret $K$ - and $L$-band visibilities of Mira-type stars. With this new model, photospheric radii were revised to lower values. In particular, Perrin et al. (2004) derived the photospheric diameters of four sources of the sample of Danchi et al. (1994): Mira, R Leo, $\chi$ Cyg, and U Ori. In all cases, they were smaller by a factor 1.5 to 2.5 than those assumed by Danchi et al. (1994). As a consequence, the ratio of the dust shell radius to photospheric radius of Danchi et al. (1994) for these sources has to be revised to higher values ranging from 4 to 30 . The lowest values are reached for Mira and $\mathrm{R}$ Leo. In addition, the visibility curves of Danchi et al. (1994) ma may be contaminated by the hot molecular envelope. This contamination may bias the inner radius of the silicate dust shell toward a lower value because it increases the visibilities. This is clearly visible in our MIDI data: the apparent diameter of the sources (after normalizing the visibilities) is dominated by the molecular envelope (factor of 2 with respect to the photospheric diameter). Taking into account the molecular envelope therefore leads to revising the $3-5$ value of the dust shell to photospheric radius ratio toward higher values. We derived a new ratio from our MIDI measurements. The fact that the normalization of the MIDI visibilities with Eq. (1) worked so well demonstrates the existence of some uncoherent flux, which we attribute to a fully resolved dust shell. From this simple model, we deduce that the dust shell is fully resolved by MIDI even on the shortest baselines and at all wavelengths. An approximate smallest possible diameter for the dust shell can then be derived by dividing the longest wavelength by the shortest baseline. Using the photospheric sizes of Table 2, we deduce a smallest silicate dust shell radius of $12-16 R \star$.

\subsection{Stellar diameter and temperature estimates}

Estimates of the stellar diameter and temperature are necessary to interpret the visibilities. Only a single baseline is available for S Ser in the $K$ band, and as a consequence, there is no way to derive both the envelope and stellar characteristics, hence the necessity to have an a priori estimate of the stellar diameter and temperature. Since the envelope optical depth is always relatively high across the $N$ band, very little or even no information can be derived about the star itself from the $N$-band data, and it is also necessary to use an a priori estimate of the central star's characteristics. Ideally, one would use a diameter measured at near-infrared wavelengths where the contribution of the envelope is lower. No reliable diameter (i.e., no diameter measurements that take into account the molecular envelope) has been measured for the stars of our sample. It is not possible to derive a good diameter for $\mathrm{S}$ Ser with a single-baseline $K$-band measurement because the visibility function is too complex to be able to do so. We explored two different methods, one based on past FLUOR measurements and one based on nearinfrared photometry. Firstly, we used the $J H K$ ( $L$ in addition for $\mathrm{S}$ Ser) fluxes available from the literature (from 2MASS Cutri et al. 2003; for all sources but S Ser and from Whitelock et al. 2000, for S Ser, the pulsation phases of the MIDI and Keck 

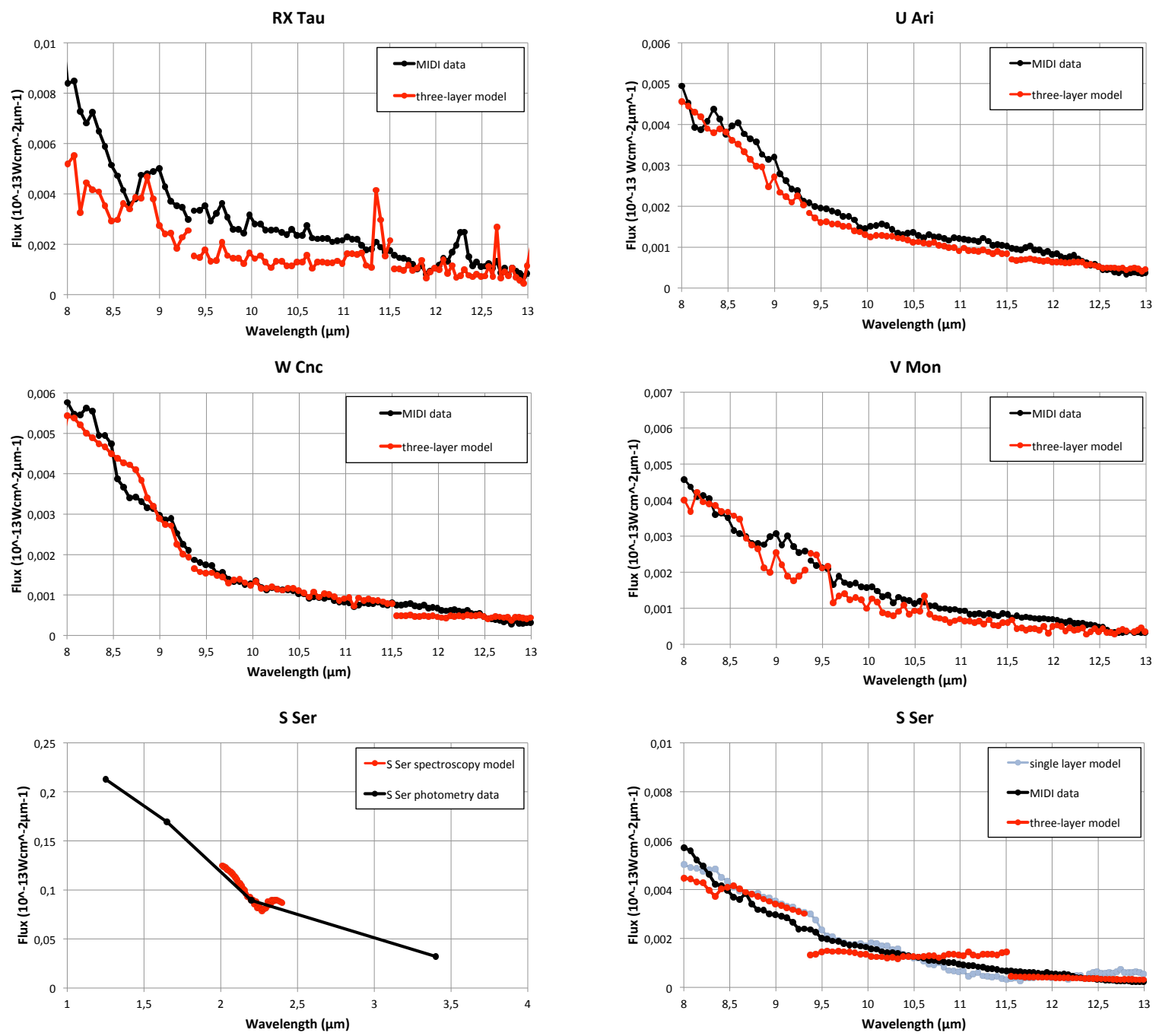

Fig. 2. Comparison between the mid-IR synthetic spectra and the MIDI calibrated spectra and between the near-IR photometry and $K$-band synthetic spectrum for S Ser. The spectra were not fitted but used as constraints to model the visibilities. The synthetic spectra for the $N$ band are based on the three-layer model, one per range of wavelengths: 7.80-9.30 $\mu \mathrm{m}, 9.37-11.50 \mu \mathrm{m}$, and 11.55-13.26 $\mu \mathrm{m}$. The light gray spectrum model for S Ser has been obtained fitting the visibilities with a single envelope.

observations were computed for S Ser and were used to interpolate the JHKL measurements; this was not possible for the 2MASS fluxes) and these fluxes were fitted with a blackbody function with the source temperature and angular diameter as free parameters. Because the near-infrared bands are mostly free of molecular absorptions (the lowest $H$ opacity is in the $H$ band), and because these wavelength ranges are close to the peak of the source SED (around $1 \mu \mathrm{m}$ ), the photometric diameter is expected to be a reasonable estimate of a physical diameter assuming the central object can be described by a simple star from a radiometric point of view. The method provides estimates with an accuracy certainly better than $10 \%$.

For the second method, we compared the central object interferometric diameter for the sample of Perrin et al. (2004) with the photometric diameter computed from the photometric magnitudes interpolated at the phase of the observations and given in the same paper. A global law $\varnothing_{\text {int }}=0.81 \times$ phot was derived, but the scatter of the distribution is quite high. As a consequence, we decided to use the photometric diameters for all sources but S Ser since no phased photometry was available and since there is no reliable way to derive a diameter with an accuracy better than $20 \%$ in practice under these conditions. For S Ser we used the photometric diameter to interpret the MIDI data and the diameter corrected with the above law to interpret the $K$-band data. The spectral types were used to determine temperatures using effective temperature scales by Perrin et al. (1998) and Ridgway et al. (1980). The central star's diameters and temperatures are listed in Table 2.

\subsection{Modeling the K-band visibilities of S Ser}

The $K$-band spectral visibilities were fitted with the star+envelope model adjusting the envelope diameter, the envelope temperature, and the envelope optical depth (Fig. 1). When data have been obtained on a single baseline, there are more parameters than data points, and the $\chi^{2}$ is usually equal to 0 . The $K$-band flux of Whitelock et al. (2000) at the closest phase when S Ser was observed was used to constrain the global adjustment of the spectrum (Fig. 2). This led to the parameters given in Table 2 for the envelope in the $K$ band and to optical depths ranging from 0.08 where the absorption by $\mathrm{CO}$ and $\mathrm{H}_{2} \mathrm{O}$ is minimum up to 0.72 at $2.4 \mu \mathrm{m}$ where the absorption is 
Table 2. Results of infrared visibility modeling.

\begin{tabular}{|c|c|c|c|c|c|c|c|c|c|}
\hline Star & Band & Spectral type & $\begin{array}{c}\emptyset_{\star} \\
\text { (mas) }\end{array}$ & $\begin{array}{c}\emptyset_{1900 \mathrm{~K}} \\
\text { (mas) }\end{array}$ & $\begin{array}{c}\emptyset_{\text {silicate }}^{\text {MIDI }} \\
\text { (mas) }\end{array}$ & $\begin{array}{l}T_{\star}^{\mathrm{eff}} \\
(\mathrm{K})\end{array}$ & $\begin{array}{c}\emptyset_{\mathrm{env}} \\
\text { (mas) }\end{array}$ & $\begin{array}{l}T_{\text {env }} \\
(\mathrm{K})\end{array}$ & $\chi^{2}$ \\
\hline U Ari & $\begin{array}{l}7.80-9.30 \mu \mathrm{m} \\
9.37-11.50 \mu \mathrm{m} \\
11.55-13.26 \mu \mathrm{m}\end{array}$ & M5.5 III & 5.00 & 15.08 & 62.3 & $3300^{a}$ & $\begin{array}{l}15.75 \\
17.16 \\
21.67\end{array}$ & $\begin{array}{c}2800 \\
1330 \\
750\end{array}$ & $\begin{array}{l}26.98 \\
34.37 \\
10.27\end{array}$ \\
\hline W Cnc & $\begin{array}{l}7.80-9.30 \mu \mathrm{m} \\
9.37-11.50 \mu \mathrm{m} \\
11.55-13.26 \mu \mathrm{m}\end{array}$ & M7 III & 3.90 & 10.29 & 43.4 & $3087^{a}$ & $\begin{array}{l}13.00 \\
12.00 \\
16.00\end{array}$ & $\begin{array}{c}1600 \\
1150 \\
700\end{array}$ & $\begin{array}{l}5.63 \\
3.48 \\
10.53\end{array}$ \\
\hline RX Tau & $\begin{array}{l}7.80-9.30 \mu \mathrm{m} \\
9.37-11.50 \mu \mathrm{m} \\
11.55-13.26 \mu \mathrm{m}\end{array}$ & M7e & 5.38 & 14.20 & 69.4 & $3087^{a}$ & $\begin{array}{l}13.00 \\
21.88 \\
28.00\end{array}$ & $\begin{array}{l}1900 \\
1875 \\
1800\end{array}$ & $\begin{array}{l}57.34 \\
96.74 \\
143.04\end{array}$ \\
\hline V Mon & $\begin{array}{l}7.80-9.30 \mu \mathrm{m} \\
9.37-11.50 \mu \mathrm{m} \\
11.55-13.26 \mu \mathrm{m}\end{array}$ & M6e & 3.89 & 11.33 & 62.1 & $3243^{a}$ & $\begin{array}{l}10.75 \\
10.00 \\
10.00\end{array}$ & $\begin{array}{l}1800 \\
2000 \\
2000\end{array}$ & $\begin{array}{l}0.08 \\
0.04 \\
58.94\end{array}$ \\
\hline S Ser & $\begin{array}{l}\mathrm{K} \\
7.80-9.30 \mu \mathrm{m} \\
9.37-11.50 \mu \mathrm{m} \\
11.55-13.26 \mu \mathrm{m}\end{array}$ & M5e & $\begin{array}{l}3.20 \\
3.60\end{array}$ & 10.37 & - & $3420^{b}$ & $\begin{array}{l}7.00 \\
10.50 \\
15.00 \\
19.50\end{array}$ & $\begin{array}{c}2000 \\
2000 \\
1750 \\
500\end{array}$ & $\begin{array}{l}- \\
- \\
- \\
-\end{array}$ \\
\hline RT Aql & - & M7e & 3.38 & 8.92 & - & $3087^{a}$ & - & - & - \\
\hline
\end{tabular}

Notes. $\varnothing_{\star}$ is the estimated photospheric diameter with an accuracy better than $10 \%$ (except for S Ser, for which it is about $20 \%$ ). $\emptyset_{1900 \mathrm{~K}}$ is twice the estimated condensation radius of $\mathrm{Al}_{2} \mathrm{O}_{3} . \varnothing_{\text {silicate }}^{\mathrm{MIDI}}$ is the estimated smallest possible diameter of the silicate dust shell. $T_{\star}^{\text {eff }}$ is the stellar effective temperature estimated from photometric measurements, and $\emptyset_{\star} . \varnothing_{\mathrm{env}}$ and $T_{\mathrm{env}}$ are the diameter and temperature of the envelope above the photosphere in the simple star+envelope model. Uncertainties of these parameters were not analyzed in this paper, the values are therefore indicative. The last column gives the reduced $\chi^{2}$ of the fit. No value is given for S Ser because only one baseline was measured and there are fewer data points than model parameters. ${ }^{(a)}$ Interpolated from Perrin et al. (1998); ${ }^{(b)}$ interpolated from Ridgway et al. (1980).

maximum. The envelope/stellar diameter ratio is about 2.2 , as was found in Perrin et al. (2004).

\subsection{Modeling the $\mathrm{N}$-band visibilities}

The same procedure was used for the $N$-band data. The $N$-band spectra calibrated as explained in Sect. 3.2 were used to bring an additional constraint, which proved to be very important. It was not possible in general to convincingly fit both visibilities and spectra with a single set of parameters for the whole $N$ band. This was also shown by Ohnaka (2004), who needed at least two water vapor layers to fit mid-infrared data. The direct conclusion is that the molecular environment cannot be described by a single envelope, but only by an envelope with several layers whose opacities vary with wavelength. We decided to fit the data with a discrete number of layers, more exactly, with three layers that reflect three molecules used by various authors for AGB and RSG stars (e.g., Fig. 7 of Perrin et al. 2007): SiO vapor, water vapor, and alumina. Alumina has been detected in all the stars of our sample with IRAS (Onaka et al. 1989). We defined one layer for each of the following domains: 7.80-9.30 $\mu \mathrm{m}$ ( $\mathrm{SiO}$ vapor region), 9.37-11.50 $\mu \mathrm{m}\left(\mathrm{Al}_{2} \mathrm{O}_{3}\right.$ and $\mathrm{H}_{2} \mathrm{O}$ vapor region), and 11.55-13.26 $\mu \mathrm{m}$ (water vapor band region). We decided to assign the $\mathrm{Al}_{2} \mathrm{O}_{3}$ subscript to the medium range only because the opacity of alumina as computed by Verhoelst et al. (2006) peaks in this range, whereas the opacity of $\mathrm{H}_{2} \mathrm{O}$ is higher in the upper range. The visibility fits are presented in Fig. 1, the synthetic model is compared to the calibrated spectra in Fig. 2. The best-fit model parameters derived for each source are listed in Table 2. Although only one baseline was available for S Ser, one of the $\chi^{2}$ values is different from 0 : the model parameters are not degenerate in this case. We are aware that the names of the regions may sound arbitrary because no spectroscopic analysis has been performed. This is of course based on conclusions of previous works.

\subsection{Discussion of modeling results}

We first note that the model by Perrin et al. (2004) is a very simple model designed to explore the envelope around Mira stars, not to describe the exact nature of the envelope for a given star. The envelope parameters listed in Table 2 are therefore indicative, and no uncertainty analysis has been performed. Except for S Ser, for which only one baseline was available for both $K$ and $N$-band measurements, for all other sources $\chi^{2}$ values are indicative of the quality of the fits. The $\chi^{2}$ is usually higher in the $11.55-13.26 \mu \mathrm{m}$ sub-band than in other sub-bands probably because the structure of the envelope is more complex: water maybe present at different altitudes and farther away, which may explain the larger radii as shown in comparisons between nearinfrared data and dynamical model atmospheres (see, e.g., result on S Orionis by Wittkowski et al. 2008). RX Tau seems to be a particular case with quite high $\chi^{2}$ values and a larger spread in envelope diameter values that indicates a more extended envelope contributing at a wider range of distances from the star. The ratio between envelope and stellar radii at the shortest wavelength range agrees with the findings of Perrin et al. (2004), indicating that the same regions are sampled as in the nearinfrared, which is not the case at longer wavelengths. For U Ari and $\mathrm{W}$ Cnc the envelope-to-star diameter ratio is always higher than what is measured in the near-infrared, either because the envelope thickens at higher altitude in the mid-infrared, or because 
Table 3. Observed $\mathrm{SiO}$ masers.

\begin{tabular}{lcrc}
\hline \hline Star & Session $^{a}$ & $\begin{array}{r}\text { Vel } \\
\left(\mathrm{km} \mathrm{s}^{-1}\right)\end{array}$ & Detected \\
\hline U Ari & 1 & -57.0 & $\mathrm{Y}$ \\
W Cnc & 1 & 37.0 & $\mathrm{Y}$ \\
RX Tau & 1 & -41.0 & $\mathrm{Y}$ \\
X Gem & 1 & 28.0 & $\mathrm{~N}$ \\
V Mon & 1 & 5.0 & $\mathrm{Y}$ \\
RT Aql & 2 & -28.0 & $\mathrm{Y}$ \\
S Ser & 2 & 20.0 & $\mathrm{Y}$ \\
RT Oph & 2 & -40.0 & $\mathrm{~N}$ \\
T Oph & 2 & -29.0 & $\mathrm{~N}$ \\
\hline
\end{tabular}

Notes. ${ }^{(a)} 1=1$ July 2007, 2 = 24 February 2008.

the envelope structure is different from that of the sources in the sample of Perrin et al. (2004). For S Ser, the ratio in the $K$ band is the same as was found in the sample of stars with the FLUOR instrument, but the ratio is more than doubled in the mid-infrared. For $\mathrm{V}$ Mon, the ratio is indeed remarkably constant and compatible with the $K$-band ratio, pointing toward a more compact and less complex envelope than for the other sources (the $\chi^{2}$ values are generally low, another signature of a simple envelope structure). In this case, the temperature found for the envelope is even similar to the findings in $K$ band.

From these measurements the conclusion can be drawn that the mid-infrared visibilities generally probe a more extended envelope than the near-infrared ones, as was mentioned earlier by other authors (see, e.g., Ohnaka 2004). The shorter wavelengths of the $N$ band, which are sensitive to $\mathrm{SiO}$ vapor, seem to probe regions closer to the near-infrared envelope where the density is higher.

The differences between the sources in the sample may be due to different ages and could be investigated but this is beyond the scope of this paper.

\section{SiO observations and data reduction}

The observations were obtained with the NRAO Very Long Baseline Array (VLBA) during two 10-h sessions on 1 July 2007 and 24 February 2008. Two $4 \mathrm{MHz}$-wide channels in each rightand left-hand circular polarization were recorded at 42.820587 and $43.122027 \mathrm{GHz}$ to cover the $v=2, J=1-0$ and $v=1$, $J=1-0$ masing transitions of SiO. Two-bit sampling was used in the recording. The correlations resulted in 128 channels in each of the combinations of right- and left-circular polarization for each transition. A strong, nearby continuum source was observed before each star to serve as delay, bandpass, and polarization calibrator. The observations are summarized in Table 3, which lists the stars, session of observation, central velocities, and whether or not masers were detected. The calibrators are all quasars with milliarcsecond accuracy positions; the HIPPARCOS (ESA 1997) positions of the stars evaluated at the epoch of the observations were used. Note: the center velocities were picked to center the spectra in the observing band and are not necessarily the stellar systemic velocities.

Calibration and processing followed the procedure described in Cotton et al. (2004, 2006), except as noted below. Calibration was made with the NRAO AIPS package and imaging and subsequent processing with the Obit package. There is no absolute position information among all observations.
Table 4. SiO ring diameters.

\begin{tabular}{lrrrrrr}
\hline \hline Source & $\begin{array}{r}v=1^{a} \\
\text { Diam } \\
\text { mas }\end{array}$ & $\begin{array}{r}\text { Width } \\
\text { mas }\end{array}$ & Fract & $\begin{array}{r}v=2^{b} \\
\text { Diam } \\
\text { mas }\end{array}$ & $\begin{array}{r}\text { Width } \\
\text { mas }\end{array}$ & Fract \\
\hline W Cnc & 9.8 & 0.8 & 0.80 & 7.8 & 0.8 & 0.58 \\
RX Tau & 11.2 & 0.6 & 0.77 & 10.8 & 0.6 & 0.86 \\
S Ser & 9.0 & 0.7 & 0.86 & 7.2 & 0.5 & 0.82 \\
RT Aql & 14.5 & 1.0 & 0.70 & 13.7 & 0.8 & 0.75 \\
\hline U Ari & $\geq 7$ & - & - & $\geq 7$ & - & - \\
V Mon & $\geq 10$ & - & - & - & - & - \\
\hline
\end{tabular}

Notes. No ring could be fitted for U Ari and V Mon, but smallest possible diameter values are given. Fract is the fraction of the emission in the ring. ${ }^{(a)} v=1, J=1-0$ transition of $\mathrm{SiO}$ at $43.1 \mathrm{GHz} ;{ }^{(b)} v=2$, $J=1-0$ transition of $\mathrm{SiO}$ at $42.8 \mathrm{GHz}$.

\section{SiO results}

The intensity-weighted velocity images are plotted in Fig. 3. Photospheric mid-infrared sizes and $\mathrm{Al}_{2} \mathrm{O}_{3}$ condensation diameters (see Sect. 7) are superimposed.

\subsection{SiO ring diameter}

For images for which there were sufficient masers to define a ring, the diameters and widths of the maser rings were determined from a moment analysis of compressed (2D) Stokes I images in both transitions using a direct parameter search, which minimized the sum of the ring widths. The independent ring diameters and widths were determined jointly for the two transitions, but with a common center. This procedure, especially the estimated ring widths, may be adversely affected by residual errors in the alignment of the images in the two transitions. For U Ari (both transitions) and V Mon (only the $v=1$ transition), no ring could be fitted, but a smallest ring diameter was derived from the line length joining two well-detectable spots.

The fitted ring diameters, widths, and fraction of the emission appearing in this ring for each star and transition are shown in Table 4. Individual measurements are discussed below.

\subsection{Discussion of SiO measurements}

U Ari. Too few masers were detected to define the ring. Those that were detected, were confined to a spread of about $1 \mathrm{~km} \mathrm{~s}^{-1}$. Lower limits for ring diameters are defined by measuring the distance between the two main blobs.

W Cnc. The maser ring is reasonably well defined, especially on the southern edge of the star. The masers are spread over about $10 \mathrm{~km} \mathrm{~s}^{-1}$.

$\mathrm{RX}$ Tau. The maser ring is well filled in; the bulk of the masers occurs within a velocity spread of about $7 \mathrm{~km} \mathrm{~s}^{-1}$.

$\mathrm{V}$ Mon. The masers were only detected in the $v=1, J=$ 1-0 transition of $\mathrm{SiO}$ at $43.1 \mathrm{GHz}$ transition and were too few to define a ring. A smallest possible diameter was defined by using the two blobs visible in the $v=1$ image. The detected masers were spread over $5 \mathrm{~km} \mathrm{~s}^{-1}$. 


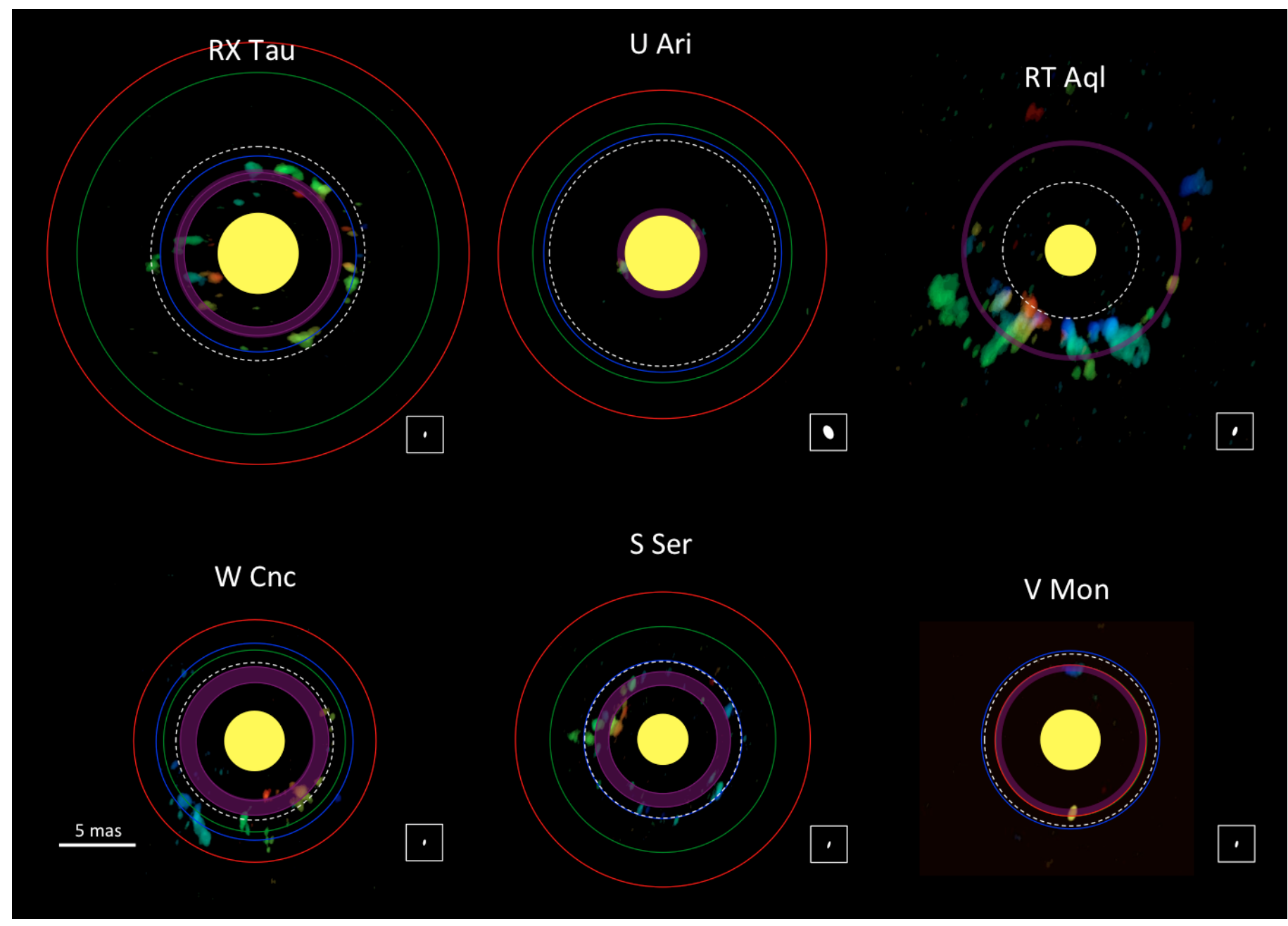

Fig. 3. SiO maser images and sizes for sources with positive maser detection. The color-coded images are intensity-weighted velocity images with red receeding and blue approaching with respect to the systemic velocity. The images are the sum of the $\mathrm{SiO} v=2, J=1-0$ transition at $42.8 \mathrm{GHz}$ and of the $v=1, J=1-0$ transition at $43.1 \mathrm{GHz}$. Sizes are superimposed. Maser image resolutions and beam orientations are given in white squares. The yellow disk is the stellar photosphere. The white dashed circle shows the condensation distance of $\mathrm{Al}_{2} \mathrm{O}_{3}$. The blue, green, and red circles are the mid-infrared sizes in the 7.80-9.30 $\mu \mathrm{m}(\mathrm{SiO}), 9.37-11.50 \mu \mathrm{m}\left(\mathrm{Al}_{2} \mathrm{O}_{3}\right.$ and $\mathrm{H}_{2} \mathrm{O}$ vapor) and 11.55-13.26 $\mu \mathrm{m}$ (water vapor) bands. The purple ring features the SiO-fitted rings. The ring could not be directly fitted to the $\mathrm{SiO}$ data for $\mathrm{V}$ Mon and $\mathrm{U}$ Ari and lowest values are shown. For V Mon, only the $v=1, J=1-0$ transition at $43.1 \mathrm{GHz}$ has been detected and is represented.

RT Aql. Maser spots are detected in the two transitions. The masers are spread over about $8 \mathrm{~km} \mathrm{~s}^{-1}$.

S Ser. Maser spots are clearly defined. The masers are spread over about $8 \mathrm{~km} \mathrm{~s}^{-1}$.

\section{Discussion}

It has been shown in earlier papers cited in the introduction that the size of Mira stars is very dependent upon wavelength. The goal of our paper is to contribute to understanding the structure of the atmosphere in relation to the process of dust formation. Molecules form in the star and are lifted higher in the atmosphere thanks to the pulsation momentum. They constitute the reservoir for dust components. Dust is detected at larger distances, but the path between molecular gas and dust is not clear yet. $\mathrm{SiO}$ masers are tracers of the $\mathrm{SiO}$ vapor. They form at a few stellar radii in the molecular envelope before $\mathrm{SiO}$ condenses into dust. The impact of dust on $\mathrm{SiO}$ masers is not completely clear, however. Gray et al. (2009) found that dust can ever play a positive or a negative role on $\mathrm{SiO}$ masers, depending on conditions, so that the link between $\mathrm{SiO}$ maser and dust formation radii is not straightforward. Our diameter measurements for a sample of stars give a view of the structure of the atmosphere and of where molecular gas is located. The direct comparison between the infrared and the $\mathrm{SiO}$ maser data indicates the atmospheric structure from which we try to understand how dust forms as a function of distance.

In addition to the measured distances, we calculated a rough estimate of the condensation radius for corundum for each source. The condensation temperature depends upon local pressure, as shown by the diagrams of Lodders \& Fegley (1999, Fig. 1) or Gail (2010, Fig. 5). The dynamical models of AGB stars by Freytag \& Höfner (2008) predict a gas pressure of 1 bar at less than 50 stellar radii from the star and a dynamical pressure of $10^{-2}$ bar in the same range of distances. We chose to set the pressure to $10^{-2}$ bar in a conservative approach because the condensation temperature increases with pressure. The above diagrams yield a temperature of $1900 \mathrm{~K}$, which is also the temperature used in Verhoelst et al. (2006). We assumed that an approximation of the condensation radius for corundum can 
be obtained by calculating the distance at which corundum dust would be heated to $1900 \mathrm{~K}$ by the star. The radiative transfer model is simple: an optically thick layer of corundum is directly exposed to the radiation of the photosphere and in thermal equilibrium. The relation between the star and the corundum layer temperatures and radii is then simple $\left(R_{\star} \times T_{\star}^{2}=R \times T^{2}\right)$ and yields the $R_{1900 \mathrm{~K}}$ radius as a function of the stellar parameters. It is plotted as a white dashed circle in Fig. 3. All diameters are listed in Table 2.

We obtained a single $K$-band diameter measurement, the one of S Ser. The $K$ diameter of the envelope for S Ser $\sim 2.2 R_{\star}$ agrees with previous results and matches the $42.8 \mathrm{GHz}$ $\mathrm{SiO}$ maser transition diameter. It is smaller than that of the other transition, which agrees with the finding that $\mathrm{SiO}$ masers trace upper layers of the atmosphere, as found in previous studies. The $N$-band diameters for S Ser are all larger than the $K$-band diameters. Water vapor contributes to both $K$ and $N$ bands (Ohnaka et al. 2005; Wittkowski et al. 2008; Eisner et al. 2007). This shows that water vapor and probably other molecules are present at various altitudes and that higher altitude layers are sampled in the $N$ band where molecules become cooler and closer to the dust condensation radius. Although no other star of our sample has been measured in $K$ and $N$, it seems correct to extend this conclusion to all other stars because the ratio between the $\mathrm{N}$-band diameters and the estimated photospheric radius is always larger or even much larger than 2 , which has been systematically found from measurements.

We find the $\mathrm{SiO}$ maser-to-photospheric size ratios to be consistent with those of other papers cited in the introduction. It is most often $R_{\mathrm{SiO}}^{\mathrm{maser}} \sim 2 R_{\star}$, except for $\mathrm{RT} \mathrm{Aql}$, for which $R_{\mathrm{SiO}}^{\text {maser }} \sim 3 R_{\star}$. But the $\mathrm{SiO}$ maser ring scatter for this source is much larger than for the others.

We broke the $N$ band into different subranges that are representative of molecules detected in interferometric $N$-band data in previous papers. We therefore defined three radii in the $N$ band: $R_{\mathrm{SiO}}^{\mathrm{MIDI}}$ for the 7.80-9.30 $\mu \mathrm{m}$ range (which includes the $\Delta v=$ $1 \mathrm{SiO}$ maser pumping band at $8.13 \mu \mathrm{m}), R_{\mathrm{Al}_{2} \mathrm{O}_{3}-\mathrm{H}_{2} \mathrm{O}}^{\mathrm{MIDI}}$ for the 9.37-11.50 $\mu \mathrm{m}$ range and $R_{\mathrm{H}_{2} \mathrm{O}}^{\mathrm{MIDI}}$ for the $11.55-13.26 \mu \mathrm{m}$ range. $R_{\mathrm{H}_{2} \mathrm{O}}^{\mathrm{MIDI}}$ is systematically the largest of all radii and exceeds the photospheric size by a factor of up to 6 . V Mon is the only source for which all three mid-IR radii are similar. For S Ser, the difference with the $K$ band is also large, almost a factor of 3. This shows that water vapor is present at high altitudes and provides a measurement of how extended the envelope of an AGB star is. The $\mathrm{MIDI} / \mathrm{SiO}$ and the $\mathrm{SiO}$ maser sizes match well with $R_{\mathrm{SiO}}^{\mathrm{MIDI}} \in[1.2,1.47] R_{\mathrm{SiO}}^{\mathrm{maser}}$. The range found for $\mathrm{U}$ Ari and V Mon (2 and 1, respectively) can be claimed to be quite consistent given the lack of $\mathrm{SiO}$ data to better define ring diameters. We therefore conclude that the MIDI/SiO and $\mathrm{SiO}$ maser data sample have about the same range of radii. It is interesting to note that the $\mathrm{MIDI} / \mathrm{SiO}$ diameter is systematically larger than the $\mathrm{SiO}$ maser ring diameter. This means that the $\mathrm{SiO}$ maser emission consistently occurs inside the $\mathrm{SiO}$ vapor envelope and mainly at the top of the envelope. Despite the simple definition of the corundum condensation distance, $R_{1900 \mathrm{~K}}$ is always slightly lower or similar to $R_{\mathrm{Al}_{2} \mathrm{O}_{3}-\mathrm{H}_{2} \mathrm{O}}^{\mathrm{MIDI}}$. The only exception is $\mathrm{RX}$ Tau, for which $R_{\mathrm{Al}_{2} \mathrm{O}_{3}-\mathrm{H}_{2} \mathrm{O}}^{\mathrm{MIDI}}$ and $R_{\mathrm{H}_{2} \mathrm{O}}^{\mathrm{MIDI}}$ are much larger than $R_{\mathrm{SiO}}^{\mathrm{MIDI}}$ probably because of a large amount of water vapor at up to 4 stellar radii from the star. In all cases, the water vapor envelope is quite extended, and the corundum tends to form closer to the star. One may think that $R_{\mathrm{Al}_{2} \mathrm{O}_{3}-\mathrm{H}_{2} \mathrm{O}}^{\mathrm{MIDI}}$ is all the closer to $R_{1900 \mathrm{~K}}$ because there is more corundum dust than water. This is very qualitative at that stage since we do not have a physical model of the envelope. Since corundum is the first dust species to condense, $R_{\mathrm{Al}_{2} \mathrm{O}_{3}-\mathrm{H}_{2} \mathrm{O}}^{\mathrm{MIDI}}$ is an indication of the upper value of the internal radius of the dust shell. We find $R_{\mathrm{Al}_{2} \mathrm{O}_{3}-\mathrm{H}_{2} \mathrm{O}}^{\mathrm{MIDI}} \sim 3-4.5 R_{\star}$, which is higher than the results of Wittkowski et al. (2007): $R_{\text {in }} \sim 2 R_{\star}$. This is consistent with the contamination of the radius measurement by water vapor. Our $R_{\mathrm{Al}_{2} \mathrm{O}_{3}}^{\mathrm{MIDI}}$ is also consistently smaller than our estimate of the smallest possible inner radius of the silicate dust shell of $12-16 R_{\star}$. Except for RT Aql, the $R_{1900 \mathrm{~K}}$ radius is always similar to or larger than the $\mathrm{SiO}$ maser radius and similar to the $R_{\mathrm{SiO}}^{\mathrm{MIDI}}$ radius. The difference is quite large in the case of RT Aql. We verified that the photospheric radius is consistent with the diameter measured by van Belle et al. (2002) in $K$ band: $7.24 \pm 0.42$ mas. It was measured at $V=84 \%$ visibility. In full $K$ band, based on measurements by Perrin et al. (2004), the size is probably essentially that of the molecular envelope, and this is compatible with the diameter of 3.38 mas computed from photometry (Perrin et al. 2004, found a factor of 2 between the molecular envelope size in $K$ and the photospheric size). The difference may not be explained by an error on effective temperature (derived from the stellar spectral type, which varies during the pulsation cycle) nor as an increase of $T_{\star}$ by $800 \mathrm{~K}$, which would be required, which is very high. RT Aql probably is a particuliar case for which much $\mathrm{SiO}$ is present in the atmosphere that cannot all be efficiently adsorbed by the corundum dust at the inner edge of the dust shell. RT Aql has the strongest detectable maser emission of all the objects in our sample. Although it is hard to deduce the amount of $\mathrm{SiO}$ from the strength of the masers, this points toward large $\mathrm{SiO}$ contents and is compatible with our hypothesis.

To summarize, a global view of the atmospheric structure is given by the following equations:

$R_{\text {silicate }}^{\mathrm{MIDI}} \geq R_{\mathrm{H}_{2} \mathrm{O}}^{\mathrm{MIDI}} \geq R_{\mathrm{Al}_{2} \mathrm{O}_{3}-\mathrm{H}_{2} \mathrm{O}}^{\mathrm{MIDI}} \geq R_{\mathrm{SiO}}^{\mathrm{MIDI}} \sim R_{1900 \mathrm{~K}} \geq R_{\mathrm{SiO}}^{\mathrm{maser}}$

$12-16 R_{\star} \quad 3-6 R_{\star} \quad 3-4.5 R_{\star} \quad 2.4-3 R_{\star} \quad 2 R_{\star}$.

\section{Conclusion}

We have measured sizes of AGB stars at different wavelengths using VLTI/MIDI, the Keck interferometer, and VLBA. The MIDI visibilities were converted to sizes of molecular envelopes using results of previous investigations. The $7.80-13.26 \mu \mathrm{m}$ range was divided into three subranges dominated by $\mathrm{SiO}$ vapor, corundum, and $\mathrm{H}_{2} \mathrm{O}$ vapor, and $\mathrm{H}_{2} \mathrm{O}$ vapor alone. Three different sizes were measured almost systematically, showing the stratification of the envelope. In addition, there is a clear correlation between the size of the $\mathrm{SiO}$ envelope measured with MIDI and the size of the $\mathrm{SiO}$ maser ring measured with VLBA. The size of what we called the corundum shell is found to be slightly larger than the estimated condensation distance, but the former measurement is biased toward higher values by the contamination from the more extended water vapor envelope because both corundum and water vapor contribute in this wavelength range.

We deduce from our measurements that there is clear evidence that while water vapor and corundum are found at larger distances, $\mathrm{SiO}$ is only found in vapor form and within 2 to $3 R^{\star}$, that is, at distances shorter than where silicate dust can condense. An interesting question is therefore what happens to $\mathrm{SiO}$ in the intermediate region between the silicate dust condensation radius and the $\mathrm{SiO}$ vapor envelope. One possibility is that $\mathrm{SiO}$ may not be detected because it is no longer present in the gaseous phase. Because of its high condensation temperature, corundum is believed to be the first dust species to condense, and it can play 
the role of nucleation site for other dust species (see Verhoelst et al. 2006 for references). As noted by Verhoelst et al., this scenario is only valid in chemical equilibrium conditions and may not apply to wind with relatively high velocities like in AGB stars. Despite this caveat, we suggest that the absence of gaseous $\mathrm{SiO}$ past the corundum condensation radius could be explained with the fact that $\mathrm{SiO}$ condenses on $\mathrm{Al}_{2} \mathrm{O}_{3}$ grains in the hightemperature atmosphere of the AGB stars of our sample. In addition, $\mathrm{SiO}$ may also have chemically disappeared after recombining with other chemical components on nucleation sites to form basic molecular components of silicate dust. Our observations are therefore fully compatible with this scenario where silicate dust can only form at large distances thanks to the action of corundum grains that serve as nucleation site. Alternatives to corundum need be investigated, however, because it is not believed to be of sufficiently high concentration to drive the wind and transport silicate dust to zones of lower temperature (Bladh \& Höfner 2012).

Acknowledgements. The authors are grateful to Omar Delaa for his participation in the reduction of the data. This research has made use of the SIMBAD and AFOEV databases, operated at CDS, Strasbourg, France. The National Radio Astronomy Observatory (NRAO) is operated by Associated Universities Inc., under cooperative agreement with the National Science Foundation.

\section{References}

Barnes, T. G., Evans, D. S., \& Moffett, T. J. 1978, MNRAS, 183, 285

Beichman, C. A., Neugebauer, G., Habing, H. J., Clegg, P. E., \& Chester, T. J. 1988, Infrared astronomical satellite (IRAS) catalogs and atlases, Vol. 1: Explanatory supplement

Bladh, S., \& Höfner, S. 2012, A\&A, 546, A76

Cohen, M., Walker, R. G., Carter, B., et al. 1999, AJ, 117, 1864

Colavita, M. M., Wizinowich, P. L., Akeson, R. L., et al. 2013, PASP, 125, 1226
Cotton, W. D., Mennesson, B., Diamond, P. J., et al. 2004, A\&A, 414, 275

Cotton, W. D., Vlemmings, W., Mennesson, B., et al. 2006, A\&A, 456, 339

Cutri, R. M., Skrutskie, M. F., van Dyk, S., et al. 2003, VizieR Online Data Catalog, II/246

Danchi, W. C., Bester, M., Degiacomi, C. G., Greenhill, L. J., \& Townes, C. H. 1994, AJ, 107, 1469

Diamond, P. J., Kemball, A. J., Junor, W., et al. 1994, ApJ, 430, L61

Eisner, J. A., Graham, J. R., Akeson, R. L., et al. 2007, ApJ, 654, L77

Engelke, C. W., Price, S. D., \& Kraemer, K. E. 2006, AJ, 132, 1445

ESA 1997, VizieR Online Data Catalog: I/239

Freytag, B., \& Höfner, S. 2008, A\&A, 483, 57

Gail, H.-P. 2010, Formation and Evolution of Minerals in Accretion Disks and Stellar Outflows, ed. T. Henning (Berlin: Springer Verlag), Lect. Notes Phys., 815,61

Gray, M. D., Wittkowski, M., Scholz, M., et al. 2009, MNRAS, 394, 51

Kervella, P., Montargès, M., Ridgway, S. T., et al. 2014, A\&A, 564, A88

Le Bouquin, J., Lacour, S., Renard, S., et al. 2009, A\&A, 496, L1

Leinert, C., Graser, U., Richichi, A., et al. 2003, The Messenger, 112, 13

Lodders, K., \& Fegley, Jr., B. 1999, in Asymptotic Giant Branch Stars, eds. T. Le Bertre, A. Lebre, \& C. Waelkens, IAU Symp., 191, 279

Mennesson, B., Perrin, G., Chagnon, G., et al. 2002, ApJ, 579, 446

Ohnaka, K. 2004, A\&A, 424, 1011

Ohnaka, K., Bergeat, J., Driebe, T., et al. 2005, A\&A, 429, 1057

Onaka, T., de Jong, T., \& Willems, F. J. 1989, A\&A, 218, 169

Perrin, G., Coude Du Foresto, V., Ridgway, S. T., et al. 1998, A\&A, 331, 619

Perrin, G., Ridgway, S. T., Mennesson, B., et al. 2004, A\&A, 426, 279

Perrin, G., Verhoelst, T., Ridgway, S. T., et al. 2007, A\&A, 474, 599

Reid, M. J., \& Menten, K. M. 1997, ApJ, 476, 327

Reid, M. J., \& Moran, J. M. 1981, ARA\&A, 19, 231

Ridgway, S. T., Joyce, R. R., White, N. M., \& Wing, R. F. 1980, ApJ, 235, 126

Tsuji, T. 2000, ApJ, 538, 801

van Belle, G. T., Thompson, R. R., \& Creech-Eakman, M. J. 2002, AJ, 124, 1706

Verhoelst, T., Decin, L., van Malderen, R., et al. 2006, A\&A, 447, 311

Whitelock, P., Marang, F., \& Feast, M. 2000, MNRAS, 319, 728

Wittkowski, M., Boboltz, D. A., Ohnaka, K., Driebe, T., \& Scholz, M. 2007, A\&A, 470, 191

Wittkowski, M., Boboltz, D. A., Driebe, T., et al. 2008, A\&A, 479, L21 